\title{
Robust Speed Tracking Control for a Micro Turbine as a Distributed Energy Resource via Feedback Domination and Disturbance Observer
}

\author{
Ancheng $X u^{1}$ and Hui Chen ${ }^{2}$ \\ ${ }^{1}$ Changzhou Institute of Technology, Changzhou, Jiangsu 213032, China \\ ${ }^{2}$ College of Automation Engineering, Shanghai University of Electric Power, Shanghai 200090, China
}

Correspondence should be addressed to Hui Chen; chenhui@shiep.edu.cn

Received 1 June 2017; Revised 3 August 2017; Accepted 14 November 2017; Published 14 December 2017

Academic Editor: J.-C. Cortés

Copyright (c) 2017 Ancheng Xu and Hui Chen. This is an open access article distributed under the Creative Commons Attribution License, which permits unrestricted use, distribution, and reproduction in any medium, provided the original work is properly cited.

Micro turbine (MT) is characterized with complex dynamics, parameter uncertainties, and variable working conditions. In this paper, a novel robust controller is investigated for a single-shaft micro turbine as a distributed energy resource by integrating a feedback domination control technique and a feedforward disturbance compensation. An active estimation process of the mismatched disturbances is firstly enabled by constructing a disturbance observer. Secondly, we adopt a feedback domination technique, rather than popularly used feedback linearization methods, to handle the system nonlinearities. In an explicit way, the composite controllers are then derived by recursive design based on Lyapunov theory while a global input-to-state stability can be guaranteed. Abundant comparison simulation results are provided to demonstrate the effectiveness of the proposed scheme, which not only perform an improved closed-loop control performance comparing to all existing results, but also render a simple control law which will ease its practical implementation.

\section{Introduction}

With fast response and high efficiency feature, micro turbine (MT) is considered to be one of the most ideal candidates to be included in the hybrid renewable energy generation systems, such as maintaining the customs side power requirement by coping with peaking demand [1-4]. It is also well known that MT system employs complex dynamics, bringing about technical challenge in control design and stability analysis. Proportional-integral methodology is generally utilized to control the output speed of the MT system [4-6], with fewer novel controller designed based on the improved model constructed in work [7].

Noticing that the nonlinearities and disturbances are inevitable in the MT system, several nonlinear controllers [710 ] and so forth are designed to control the output speed of the MT system. In the work [8], the speed control loop adopts an artificial neural network, which refers a better load following performance, but the mechanical power output experiences a momentary delay with the rotor speed decreasing/increasing. On the other hand, most of the existing results can be regarded as an "inactive" antidisturbance approach. An opposite approach, namely, "active" antidisturbance controller, which adopts a disturbance estimation part to observe a feedforward compensation, has been utilized in practical plants for years and more effective disturbance rejection performances can be realized; see [11-13] among others, for more details.

Among existing active disturbance attenuation methods, a common way applied in many of the literatures is to synthesize the load disturbance, parameter perturbations, and the hard-handling nonlinearities from the system as a lumped unknown information, which is then totally figured out by a disturbance estimation process [11, 12, 14]. In [14], the load disturbance of the MT system can be automatically restrained by the disturbance rejection control with extended state observer with a small overshoot, while it brings a longer response time. One evident drawback of this method 
is that the nominal control performance will be sacrificed with no doubt. New trends reveal that, by precisely handling system's nonlinearities, uncertainties, perturbations, and disturbances in different loops will provide an upgrade to a more delicate control and disturbance attenuation performance [15].

Feedback domination technique, rather than the popular backstepping approach, refers a more simple controller form which facilitates the practical implementations. When adopting a backstepping feedback method, the complexity and costly calculations of the controller should be conquered, such as control methods referred in [7]. In [13], an feedback domination method is applied to condense the design process and generate a more brief control form. An output feedback domination methodology is proposed in [16], which provides a simple and general control law for the low-triangle system. Furthermore, an active disturbance attenuation control for permanent magnet synchronous motor (PMSM) via feedback domination and disturbance observer proposed in [17], which refers to a simple and effective controller when system, is under perturbations. In this paper, we reinvestigate the active disturbance attenuation control research for MT system by incorporating the feedback domination technique with disturbance observer based control approach, where an exquisite handling procedure for the nonvanishing nonlinearities is also proposed.

It is noticed that, in this paper, that the studied system, control objective, and corresponding control process are all different from [17]. Meanwhile, the researched disturbance and settled working condition are also individual in the MT system. Furthermore, the controller designed for MT system in this paper has proved its global stability based on Lyapunov theory, while the controller proposed in [17] is only designed under a less ambitious semiglobal control objective. This point is essentially different owing to the fact that large-signal stability is much demanded in MT system.

The system's interior nonlinearities can be utilized in the feedback part due to the domination function, rather than cancelation. The constructed nonlinear disturbance observer will estimate both the variable and fixed perturbations in the system and then the observed messages are compensated via feedforward channels in each step's design. Hence a more brief controller expression for the MT system can be deduced. In order to show its theoretical justification, rigorous Lyapunov function based stability analysis is provided. Moreover, abundant simulation verification and comparison results are included to verify the improved performance and disturbance rejection ability. Compared with related existing results including the one in [17], the main contributions of this manuscript are listed as follows:

(i) Active disturbance attenuation method has been applied into the MT system the first time. It is of significance by noting that a high precision control objective is highly demanded in these kinds of applications.

(ii) A global active disturbance attenuation controller integrating with the domination feedback technique has been designed. Moreover, both the discrete-time and continuous-time controller forms have been presented in the manuscript.

(iii) To better demonstrate the effectiveness of the proposed method, performance comparisons of the MT system among PI controller, output feedback domination controller, four-loop PI controller, four-loop feedback domination controller, and the proposed controller are given. As a prerequisite, the simulations considered varied control objectives and complexed disturbances, which intentionally reflect the practical situation of the MT system.

The rest of the paper is organized as follows. The mathematical model of the MT system and problem formulation and several essential preliminaries are described in Section 2. In Section 3, the design of the proposed active disturbance attenuation controller is presented. The effectiveness and priority of this novel controller for MT system are proved via numerical simulations in Section 4. A conclusion and a reference list end the paper.

\section{Preliminaries and Problem Statements}

2.1. Preliminaries. For convenience of readers, several lemmas which will be utilized latter are stated as follows. Consider the following system:

$$
\dot{x}=\eta(t, x, u(t)), \quad x \in \mathbb{R}^{n}, u \in \mathbb{R}^{m}
$$

where $\eta(\cdot)$ is a continuous function with $\eta(0)=0$.

Definition 1. A continuous function $\alpha:[0, a) \rightarrow[0, \infty)$ belongs to class $\mathscr{K}$ if it is strictly increasing and $\alpha(0)=0$. It belongs to class $K_{\infty}$ if $a=\infty$ and $\alpha(r) \rightarrow \infty$ as $r \rightarrow \infty$.

Lemma 2 (see [18]). Let $V:[0, \infty) \times \Gamma_{1} \rightarrow \mathbb{R}$ be a continuously differentiable function, such that

$$
\begin{aligned}
& \alpha_{1}(\|x\|) \leq V(t, x) \leq \alpha_{2}(\|x\|), \\
& \frac{\partial V}{\partial t}+\frac{\partial V}{\partial x} \eta(t, x, u(t)) \leq-\Gamma(x), \\
& \forall\|x\| \geq \alpha_{3}(\|u(t)\|)>0,
\end{aligned}
$$

where $\alpha_{1}(\cdot), \alpha_{2}(\cdot)$ are class $\mathscr{K}_{\infty}$ functions, $\alpha_{3}(\cdot)$ is a class $\mathscr{K}$ function, and $\Gamma(x)$ is a continuous positive definite function on $\mathbb{R}^{n}$. Then system (1) is globally input-to-state stable (ISS).

Lemma 3 (see [19]). Let $c, d$ be positive constants. Given any positive smooth function $\gamma(x, y)$, the following inequality holds:

$$
\begin{aligned}
|x|^{c}|y|^{d} \leq & \frac{c}{c+d} \gamma(x, y)|x|^{c+d} \\
& +\frac{d}{c+d} \gamma^{-c / d}(x, y)|y|^{c+d} .
\end{aligned}
$$

2.2. Mathematical Modeling. For the purpose of studying the whole MT system, its block diagrams are structured in [7] under some assumptions: acceleration control loop and 
temperature control loop are omitted, the rectifier side DC current keeps constant, which means the electromagnetic torque remains unchanged if the damping torque is ignored as well as the loss of rectifier.

It is noticed that, with above explanations, a simplified mathematical model of the micro turbine system is depicted by [7]:

$$
\begin{aligned}
& \dot{x}_{1}=\frac{1.3}{J} x_{2}-\frac{1}{2 J} x_{1}+\frac{0.201-T_{e}}{J} \\
& \dot{x}_{2}=\frac{1}{T_{\mathrm{CD}}} x_{3}-\frac{1}{T_{\mathrm{CD}}} x_{2} \\
& \dot{x}_{3}=\frac{K_{a}}{T_{a}} x_{4}-\frac{1}{T_{a}} x_{3} \\
& \dot{x}_{4}=\frac{c k_{f}}{a} x_{1} u-\frac{b}{a} x_{4}+\frac{c k_{l}}{a} \\
& y=x_{1},
\end{aligned}
$$

where $x_{1}$ is the turbine speed as the output whose objective is to track an expected speed $y_{r}, J$ is the combined inertia of rotor and load, $T_{e}$ is the electromagnetic torque, $T_{\mathrm{CD}}$ is the discharge lag time constant, $K_{a}$ and $T_{a}$ are the fuel actuator parameters, $a, b$, and $c$ are the valve position parameters, $k_{l}$ is the minimum load, and $k_{f}$ is the maximum fuel demand.

2.3. Problem Formulation. Taking the external disturbances and parameters uncertainties of system (4) into consideration, (4) can be formulated into the strict-feedback form as

$$
\begin{aligned}
\dot{x}_{i} & =g_{i} x_{i+1}+f_{i}\left(\bar{x}_{i}\right)+d_{i}(t), \quad i=1,2,3 \\
\dot{x}_{4} & =g_{4}(x) u+f_{4}\left(\bar{x}_{4}\right)+d_{4}(t) \\
y & =x_{1},
\end{aligned}
$$

where $x=\left[x_{1}, x_{2}, x_{3}, x_{4}\right]^{T}$ is chosen as state variable vector, $g_{1}=1.3 / J, g_{2}=1 / T_{\mathrm{CD}}, g_{3}=K_{a} / T_{a}$, and $g_{4}(x)=\left(c k_{f} / a\right) x_{1}$ denote the coefficients, $f_{1}\left(\bar{x}_{1}\right)=(-1 / 2 J) x_{1}+\left(0.201-T_{e}\right) / J$, $f_{2}\left(\bar{x}_{2}\right)=\left(-1 / T_{\mathrm{CD}}\right) x_{2}, f_{3}\left(\bar{x}_{3}\right)=\left(-1 / T_{a}\right) x_{3}$, and $f_{4}\left(\bar{x}_{4}\right)=$ $(-b / a) x_{4}+c k_{l} / a$ denote variable functions and constant terms, $d_{i}, i=1,2,3,4$ can be regarded as lumped mismatched disturbances, and $d(t)=\left[d_{1}(t), d_{2}(t), d_{3}(t), d_{4}(t)\right]^{T}$.

Assumption 4. There exist two positive constants $\bar{d}, \widetilde{d}$ such that the following relation holds:

$$
\begin{aligned}
& d(t) \in \Gamma_{d} \triangleq\left\{d(t) \mid \sup _{t \geq 0}\|d(t)\| \leq \bar{d}\right\}, \\
& \dot{d}(t) \in \Gamma_{\dot{d}} \triangleq\left\{\dot{d}(t) \mid \sup _{t \geq 0}\|\dot{d}(t)\| \leq \widetilde{d}\right\} .
\end{aligned}
$$

Remark 5. The parameters of the micro turbine are mainly influenced by the humidity and the temperature in practical MT systems. Hence the deviations of the interior parameters are changed in a finite scale. The electromagnetic torque $T_{e}$ and the combined inertia $J$ are also limited by their nominal values. Therefore, the lumped disturbance vector $d(t)$ and its derivative are all bounded.

The objective of this paper is to design an active disturbance attenuation controller based on disturbance observer and feedback domination technique for system (5) under Assumption 4. More concisely, the control problem can be formulated as finding a speed tracking controller of the form

$$
\begin{aligned}
& \widehat{d}=\phi(\lambda, x, \widehat{d}), \\
& u=u(k, x, \widehat{d}),
\end{aligned}
$$

with suitable constants $\lambda$ and $k$, such that for any trajectories of the closed-loop system (5)-(7) can be rendered globally ISS.

\section{Main Result}

In this section, the control problem for system (5) with mismatched uncertainties/disturbances will be solved by designing a composite recursive controller through an explicit stepby-step construction procedure.

Defining $\widehat{d}_{i}(t)$ as the estimation value of $d_{i}(t)$, the form of a nonlinear disturbance observer for system (5) is described as

$$
\begin{aligned}
& \dot{p}_{i}=g_{i} x_{i+1}+f_{i}\left(\bar{x}_{i}\right)+\widehat{d}_{i}(t), \quad i=1,2,3, \\
& \dot{p}_{4}=g_{4}(x) u+f_{4}\left(\bar{x}_{4}\right)+\widehat{d}_{4}(t), \\
& \hat{d}_{i}=\lambda_{i}\left(x_{i}-p_{i}\right), \quad i=1,2,3,4,
\end{aligned}
$$

where $\lambda_{i}$ s are positive constants to be determined later. Defining

$$
e_{i}=d_{i}-\widehat{d}_{i}
$$

the disturbance estimation error system is governed by

$$
\dot{e}_{i}=-\lambda_{i} e_{i}+\dot{d}_{i}
$$

Step 1. Choose a Lyapunov function as

$$
V_{1}=\frac{1}{2} z_{1}^{2}+\frac{1}{2} e_{1}^{2}
$$

where $z_{1}=x_{1}-y_{r}$. Taking derivatives of both sides of (11) yields

$$
\dot{V}_{1}=z_{1}\left(g_{1} x_{2}+f_{1}\left(x_{1}\right)+d_{1}(t)\right)-\lambda_{1} e_{1}^{2}+\dot{d}_{1} e_{1} .
$$

With Lemma 3, the following inequalities hold:

$$
\begin{aligned}
z_{1} e_{1} & \leq \frac{1}{2} z_{1}^{2}+\frac{1}{2} e_{1}^{2}, \\
\dot{d}_{1} e_{1} & \leq \frac{1}{2} \dot{d}_{1}^{2}+\frac{1}{2} e_{1}^{2}, \\
\left|z_{1}\left(f_{1}\left(x_{1}\right)-\widehat{f}_{1}\right)\right| & \leq c_{1}\left|z_{1}\right|\left|x_{1}-y_{r}\right| \leq c_{1} z_{1}^{2},
\end{aligned}
$$

where $\widehat{f}_{1}=f_{1}\left(y_{r}\right)$ and $c_{1}$ is a positive constant. 
Utilizing the disturbance estimation value $\widehat{d}_{1}$, the virtual control law $x_{2}^{*}$ can be presented as

$$
x_{2}^{*}=-\frac{1}{g_{1}}\left(k_{1} z_{1}+\widehat{f}_{1}+\widehat{d}_{1}(t)\right),
$$

where $k_{1} \geq c_{1}+9 / 2$.

Let $z_{2}=x_{2}-x_{2}^{*}$. Then (14) can be concluded as

$$
\dot{V}_{1} \leq-4 z_{1}^{2}-\left(\lambda_{1}-1\right) e_{1}^{2}+\frac{1}{2} \dot{d}_{1}^{2}+g_{1} z_{1} z_{2} .
$$

Step 2. Choose a Lyapunov function as

$$
V_{2}=V_{1}+\frac{1}{2} z_{2}^{2}+\frac{1}{2} e_{2}^{2}
$$

Taking derivatives of both sides of (16) yields

$\dot{V}_{2}$

$$
\begin{aligned}
\leq & -4 z_{1}^{2}-\left(\lambda_{1}-1\right) e_{1}^{2}+\frac{1}{2} \dot{d}_{1}^{2}+g_{1} z_{1} z_{2} \\
& +z_{2}\left(g_{2} x_{3}+f_{2}\left(\bar{x}_{2}\right)+d_{2}-\frac{\partial x_{2}^{*}}{\partial z_{1}} \dot{z}_{1}-\frac{\partial x_{2}^{*}}{\partial \widehat{d}_{1}} \lambda_{1} e_{1}\right) \\
& -\lambda_{2} e_{2}^{2}+\dot{d}_{2} e_{2} .
\end{aligned}
$$

With Lemma 3, one can obtain the following inequalities:

$$
\begin{aligned}
z_{2} e_{2} & \leq \frac{1}{2} z_{2}^{2}+\frac{1}{2} e_{2}^{2}, \\
\dot{d}_{2} e_{2} & \leq \frac{1}{2} \dot{d}_{2}^{2}+\frac{1}{2} e_{2}^{2}, \\
g_{1} z_{1} z_{2} & \leq \frac{1}{4} z_{1}^{2}+c_{2} z_{2}^{2},
\end{aligned}
$$

where $c_{2}$ is a positive constant. Utilizing Lemma 3 , the following relation holds:

$$
\begin{aligned}
\left|z_{2}\left(f_{2}\left(\bar{x}_{2}\right)-\widehat{f}_{2}\right)\right| & \leq \alpha_{2}\left|z_{2}\right|\left(\left|x_{1}-y_{r}\right|+\left|x_{2}-\widehat{x}_{2}\right|\right) \\
& \leq \bar{\alpha}_{2}\left|z_{2}\right|\left(\left|z_{1}\right|+\left|z_{2}\right|\right) \\
& \leq \frac{1}{2} z_{1}^{2}+\check{c}_{2} z_{2}^{2}
\end{aligned}
$$

where $\widehat{f}_{2}=f_{2}\left(y_{r}, \widehat{x}_{2}\right), \widehat{x}_{2}=-\left(\widehat{f}_{1}+\widehat{d}_{1}(t)\right) / g_{1}$, and $\alpha_{2}, \bar{\alpha}_{2}, \check{c}_{2}$ are positive constants.

For briefness and consistency of the designing process, the following relation holds:

$$
\begin{aligned}
& \left|z_{2} \frac{\partial x_{2}^{*}}{\partial z_{1}} \dot{z}_{1}\right| \\
& \quad=\left|z_{2} \frac{\partial x_{2}^{*}}{\partial z_{1}}\left(g_{1} z_{2}-k_{1} z_{1}+f_{1}\left(x_{1}\right)-\widehat{f}_{1}+e_{1}\right)\right| \\
& \quad \leq\left|-z_{2} \frac{k_{1}}{g_{1}}\right|\left(\left|g_{1} z_{2}\right|+k_{1}\left|z_{1}\right|+\overline{\alpha_{1}}\left|z_{1}\right|+\left|e_{1}\right|\right) \\
& \quad \leq \frac{1}{4} z_{1}^{2}+\frac{1}{2} e_{1}^{2}+\bar{c}_{2} z_{2}^{2},
\end{aligned}
$$

With relation (14) and applying Lemma 3, the inequality can be derived as

$$
\left|z_{2} \frac{\partial x_{2}^{*}}{\partial \widehat{d}_{1}} \lambda_{1} e_{1}\right| \leq \frac{1}{2} e_{1}^{2}+\widetilde{c}_{2} z_{2}^{2},
$$

where $\widetilde{c}_{2}>0$ is a constant which will be chosen later.

Using the disturbance estimation value $\widehat{d}_{2}$, the virtual control law $x_{3}^{*}$ can be designed as

$$
x_{3}^{*}=-\frac{1}{g_{2}}\left(k_{2} z_{2}+\widehat{f}_{2}+\widehat{d}_{2}(t)\right),
$$

where $k_{2} \geq c_{2}+\bar{c}_{2}+\check{c}_{2}+\widetilde{c}_{2}+7 / 2$.

Let $z_{3}=x_{3}-x_{3}^{*}$ and substituting (22) into (17), one can conclude the following inequality as

$$
\begin{aligned}
\dot{V}_{2} \leq & -3\left(z_{1}^{2}+z_{2}^{2}\right)-\left(\lambda_{2}-1\right) e_{2}^{2}-\left(\lambda_{1}-2\right) e_{1}^{2}+\frac{1}{2} \dot{d}_{1}^{2} \\
& +\frac{1}{2} \dot{d}_{2}^{2}+g_{2} z_{2} z_{3} .
\end{aligned}
$$

Step 3. Choose a Lyapunov function as

$$
V_{3}=V_{2}+\frac{1}{2} z_{3}^{2}+\frac{1}{2} e_{3}^{2}
$$

Taking derivatives of both sides of (24) yields

$$
\begin{aligned}
\dot{V}_{3} & \leq-3\left(z_{1}^{2}+z_{2}^{2}\right)-\left(\lambda_{2}-1\right) e_{2}^{2}-\left(\lambda_{1}-2\right) e_{1}^{2}+\frac{1}{2} \dot{d}_{1}^{2} \\
& +\frac{1}{2} \dot{d}_{2}^{2}+g_{2} z_{2} z_{3}+z_{3}\left(g_{3} x_{4}+f_{3}\left(\bar{x}_{3}\right)+d_{3}\right. \\
& \left.-\sum_{j=1}^{2} \frac{\partial x_{3}^{*}}{\partial z_{j}} \dot{z}_{j}-\sum_{j=1}^{2} \frac{\partial x_{3}^{*}}{\partial \widehat{d}_{j}} \lambda_{j} e_{j}\right)-\lambda_{3} e_{3}^{2}+\dot{d}_{3} e_{3} .
\end{aligned}
$$

With Lemma 3, one can acquire the following relation:

$$
\begin{aligned}
z_{3} e_{3} & \leq \frac{1}{2} z_{3}^{2}+\frac{1}{2} e_{3}^{2}, \\
\dot{d}_{3} e_{3} & \leq \frac{1}{2} \dot{d}_{3}^{2}+\frac{1}{2} e_{3}^{2}, \\
g_{2} z_{2} z_{3} & \leq \frac{1}{4} z_{2}^{2}+c_{3} z_{3}^{2},
\end{aligned}
$$

where $c_{3}=g_{2}^{2}$.

With Lemma 3, the following inequalities hold:

$$
\begin{aligned}
& \left|z_{3}\left(f_{3}\left(\bar{x}_{3}\right)-\widehat{f}_{3}\right)\right| \\
& \quad \leq \alpha_{3}\left(\bar{x}_{3}\right)\left|z_{3}\right|\left(\left|x_{1}-y_{r}\right|+\left|x_{2}-\widehat{x}_{2}\right|+\left|x_{3}-\widehat{x}_{3}\right|\right) \\
& \quad \leq \bar{\alpha}_{3}\left(\bar{x}_{3}\right)\left|z_{3}\right|\left(\left|z_{1}\right|+\left|z_{2}\right|+\left|z_{3}\right|\right) \\
& \quad \leq \frac{1}{2} z_{1}^{2}+\frac{1}{2} z_{2}^{2}+\check{c}_{3} z_{3}^{2}
\end{aligned}
$$

where $\bar{c}_{2}>0$ is a constant which will be chosen later. 
where $\widehat{f}_{3}=f_{3}\left(y_{r}, \widehat{x}_{2}, \widehat{x}_{3}\right), \widehat{x}_{3}=-\left(\hat{f}_{2}+\widehat{d}_{2}\right) / g_{2}$, and $\check{c}_{3}$ is a positive constant.

For briefness and consistency of the deducing process, we will get the following inequality as

$$
\begin{aligned}
& \left|z_{3}\right|\left|\sum_{i=1}^{2} \frac{\partial x_{3}^{*}}{\partial z_{i}} \dot{z}_{i}\right| \\
& =\left|z_{3}\right|\left|\sum_{i=1}^{2} \frac{\partial x_{3}^{*}}{\partial z_{i}}\left(g_{i} z_{i+1}-k_{i} z_{i}+f_{i}\left(\bar{x}_{i}\right)-\widehat{f}_{i}+e_{i}\right)\right| \\
& \leq\left|z_{3}\right| \sum_{i=1}^{2} \prod_{j=i}^{2}\left|\frac{k_{j}}{g_{j}}\right|\left(\left|g_{i} z_{i+1}\right|+k_{i}\left|z_{i}\right|+\bar{\alpha}_{i}\left(\bar{x}_{i}\right)\left|z_{\mathrm{i}}\right|+\left|e_{i}\right|\right) \\
& \leq \frac{1}{2} z_{1}^{2}+\frac{1}{4} z_{2}^{2}+\frac{1}{2}\left(e_{1}^{2}+e_{2}^{2}\right)+\bar{c}_{3} z_{3}^{2},
\end{aligned}
$$

where $\bar{c}_{3}$ is a positive constant.

Integrating with relation (22) and Lemma 3 , the inequality can be deduced as

$$
\left|z_{3}\right|\left|\sum_{i=1}^{2} \frac{\partial x_{3}^{*}}{\partial \widehat{d}_{i}} \lambda_{i} e_{i}\right| \leq \frac{1}{2}\left(e_{1}^{2}+e_{2}^{2}\right)+\widetilde{c}_{3} z_{3}^{2},
$$

where $\widetilde{\mathcal{C}_{3}}$ is a positive constant.

Using the disturbance estimation value $\widehat{d}_{3}$, the virtual control law $x_{4}^{*}$ can be designed as

$$
x_{4}^{*}=-\frac{1}{g_{3}}\left(k_{3} z_{3}+\widehat{f}_{3}+\widehat{d}_{3}(t)\right),
$$

where $k_{3} \geq c_{3}+\bar{c}_{3}+\check{c}_{3}+\widetilde{c}_{3}+5 / 2$

Let $z_{4}=x_{4}-x_{4}^{*}$ and substitute (30) into (25); the following inequality can be concluded as

$$
\dot{V}_{3} \leq-2 \sum_{i=1}^{3} z_{i}^{2}-\sum_{i=1}^{3}\left(\lambda_{i}+i-4\right) e_{i}^{2}+\frac{1}{2} \sum_{i=1}^{3} \dot{d}_{i}^{2}+g_{3} z_{3} z_{4}
$$

Step 4. Choose a Lyapunov function as

$$
V_{4}=V_{3}+\frac{1}{2} z_{4}^{2}+\frac{1}{2} e_{4}^{2}
$$

Taking derivatives of both sides of (32) yields

$$
\begin{aligned}
\dot{V}_{4} & \leq-2 \sum_{i=1}^{3} z_{i}^{2}-\sum_{i=1}^{3}\left(\lambda_{i}+i-4\right) e_{i}^{2}+\frac{1}{2} \sum_{i=1}^{3} \dot{d}_{i}^{2}+g_{3} z_{3} z_{4} \\
& +z_{4}\left(g_{4}(x) u+f_{4}\left(\bar{x}_{4}\right)+d_{4}-\sum_{i=1}^{3} \frac{\partial x_{4}^{*}}{\partial z_{i}} \dot{z}_{i}\right. \\
& \left.-\sum_{i=1}^{3} \frac{\partial x_{4}^{*}}{\partial \widehat{d}_{i}} \lambda_{i} e_{i}\right)-\lambda_{3} e_{3}^{2}+\dot{d}_{4} e_{4} .
\end{aligned}
$$

With Lemma 3, one can acquire the following inequalities:

$$
\begin{aligned}
z_{4} e_{4} & \leq \frac{1}{2} z_{4}^{2}+\frac{1}{2} e_{4}^{2}, \\
\dot{d}_{4} e_{4} & \leq \frac{1}{2} \dot{d}_{4}^{2}+\frac{1}{2} e_{4}^{2}, \\
g_{3} z_{3} z_{4} & \leq \frac{1}{4} z_{3}^{2}+c_{4} z_{3}^{2},
\end{aligned}
$$

where $c_{4}=g_{3}^{2}$.

With Lemma 3 , the following inequalities hold

$$
\begin{aligned}
& \left|z_{4}\left(f_{4}\left(\bar{x}_{4}\right)-\widehat{f}_{4}\right)\right| \leq \alpha_{4}\left(\bar{x}_{4}\right)\left|z_{4}\right| \\
& \cdot\left(\left|x_{1}-y_{r}\right|+\left|x_{2}-\widehat{x}_{2}\right|+\left|x_{3}-\widehat{x}_{3}\right|+\left|x_{4}-\widehat{x}_{4}\right|\right) \\
& \quad \leq \bar{\alpha}_{4}\left(\bar{x}_{4}\right)\left|z_{4}\right|\left(\left|z_{1}\right|+\left|z_{2}\right|+\left|z_{3}\right|+\left|z_{4}\right|\right) \leq \frac{1}{2} z_{1}^{2}+\frac{1}{2} \\
& \cdot z_{2}^{2}+\frac{1}{2} z_{3}^{2}+\check{c}_{4} z_{4}^{2},
\end{aligned}
$$

where $\widehat{f}_{4}=f_{4}\left(y_{r}, \widehat{x}_{2}, \widehat{x}_{3}, \widehat{x}_{4}\right), \widehat{x}_{4}=-\left(\widehat{f}_{3}+\widehat{d}_{3}\right) / g_{3}$, and $\check{c}_{4}$ is a positive constant.

For briefness and consistency of the deducing process, the following inequality holds:

$$
\begin{aligned}
& \left|z_{4}\right|\left|\sum_{i=1}^{3} \frac{\partial x_{4}^{*}}{\partial z_{i}} \dot{z}_{i}\right| \\
& =\left|z_{4}\right|\left|\sum_{i=1}^{3} \frac{\partial x_{4}^{*}}{\partial z_{i}}\left(g_{i} z_{i+1}-k_{i} z_{i}+f_{i}\left(\bar{x}_{i}\right)-\widehat{f}_{i}+e_{i}\right)\right| \\
& \leq\left|z_{4}\right| \sum_{i=1}^{3} \prod_{j=i}^{3}\left|\frac{k_{j}}{g_{j}}\right|\left(\left|g_{i} z_{i+1}\right|+k_{i}\left|z_{i}\right|+\bar{\alpha}_{i}\left(\bar{x}_{i}\right)\left|z_{i}\right|+\left|e_{i}\right|\right) \\
& \leq \frac{1}{2} z_{1}^{2}+\frac{1}{2} z_{2}^{2}+\frac{1}{4} z_{3}^{2}+\frac{1}{2}\left(e_{1}^{2}+e_{2}^{2}+e_{3}^{2}\right)+\bar{c}_{4} z_{4}^{2},
\end{aligned}
$$

where $\bar{c}_{4}$ is a positive constant.

With relation (30) and applying Lemma 3 , the inequality can be deduced as

$$
\left|z_{4}\right|\left|\sum_{i=1}^{3} \frac{\partial x_{4}^{*}}{\partial \widehat{d}_{i}} \lambda_{i} e_{i}\right| \leq \frac{1}{2}\left(e_{1}^{2}+e_{2}^{2}+e_{3}^{2}\right)+\widetilde{c}_{4} z_{4}^{2},
$$

where $\widetilde{c}_{4}$ is a positive constant.

Utilizing the disturbance estimation value $\widehat{d}_{4}$, the control law $u$ is designed as

$$
u=-\frac{1}{g_{4}(x)}\left(k_{4} z_{4}+\widehat{f}_{4}+\widehat{d}_{4}(t)\right),
$$

where $k_{4} \geq c_{4}+\bar{c}_{4}+\check{c}_{4}+\widetilde{c}_{4}+3 / 2$.

Now the main proof of this paper is revealed.

Theorem 6. Assume Assumption 4 is satisfied in system (5). The closed-loop system containing system (5), the disturbance 
observer system (8), and the proposed controller (38) can be rendered globally ISS provided that $\lambda_{1} \geq 5, \lambda_{2} \geq 4, \lambda_{3} \geq 3$, and $\lambda_{4} \geq 2$.

Proof. Applying Lemma 3, then (33) becomes to

$$
\dot{V}_{4} \leq-\sum_{i=1}^{4} z_{i}^{2}-\sum_{i=1}^{4}\left(\lambda_{i}+i-5\right) e_{i}^{2}+\sum_{i=1}^{4} \frac{1}{2} \dot{d}_{i}^{2} .
$$

With the choice of $\lambda_{i}^{\prime}$ s, the following relation holds:

$$
\begin{aligned}
\dot{V}_{4} & \leq-\sum_{i=1}^{4} z_{i}^{2}+\frac{1}{2} \sum_{i=1}^{4}\left(\dot{d}_{i}^{2}-e_{i}^{2}\right)-\frac{1}{2} \sum_{i=1}^{4} e_{i}^{2} \\
& \leq-\sum_{i=1}^{4} z_{i}^{2}-\frac{1}{2} \sum_{j=1}^{4} e_{j}^{2},
\end{aligned}
$$

$$
\text { for }\left\|e_{i}\right\| \geq\left\|\dot{d}_{i}\right\|, \quad i=1,2,3,4 \text {. }
$$

By utilizing Lemma 2, it can be straightforwardly derived that the closed-loop system (5)-(8)-(38) is globally ISS. This completes the proof.

Remark 7. The proposed control strategy mainly distinguishes itself with existing methods in the following two aspects. First, a feedback domination approach is applied, yielding a simple controller expression of the form (38). Second, not only is matched disturbance considered, but also a more practical mismatched condition is studied whereas a precise tracking result is achieved.

Remark 8. Define

$$
\begin{aligned}
& x(t)=\left[\begin{array}{l}
x_{1}(t) \\
x_{2}(t) \\
x_{3}(t) \\
x_{4}(t)
\end{array}\right], \\
& p(t)=\left[\begin{array}{l}
p_{1}(t) \\
p_{2}(t) \\
p_{3}(t) \\
p_{4}(t)
\end{array}\right], \\
& A=\left[\begin{array}{cccc}
\lambda_{1} & g_{1} & 0 & 0 \\
0 & \lambda_{2} & g_{2} & 0 \\
0 & 0 & \lambda_{3} & g_{3} \\
0 & 0 & 0 & \lambda_{4}
\end{array}\right], \\
& B=\left[\begin{array}{l}
0 \\
0 \\
0 \\
g_{4}
\end{array}\right] \text {, } \\
& C=\left[\begin{array}{l}
-\lambda_{1} \\
-\lambda_{2} \\
-\lambda_{3} \\
-\lambda_{4}
\end{array}\right]
\end{aligned}
$$

Then the discrete-time form of the observer (8) can be described as

$$
\begin{aligned}
\widehat{d}_{i}\left(t_{k}\right) & =\lambda_{i}\left(x_{i}\left(t_{k}\right)-p_{i}\left(t_{k}\right)\right), \quad i=1,2,3,4, \\
p\left(t_{k+1}\right) & =D p\left(t_{k}\right)+E u\left(t_{k}\right)+F x\left(t_{k+1}\right), \\
t_{k} & =k T, k=0,1,2, \ldots,
\end{aligned}
$$

where $D=e^{C T}, E=\int_{0}^{T} e^{C s} d s B, F=\int_{0}^{T} e^{C s} d s A, T$ is the sampling period, $p\left(t_{0}\right)=0$, and $u\left(t_{0}\right)=0$.

The discrete-time control form for practical systems is given as follows:

$$
\begin{gathered}
u(t)=u\left(t_{k}\right), \\
\forall t \in\left[t_{k}, t_{k+1}\right), t_{k}=k T, k=0,1,2, \ldots, \\
u\left(t_{k}\right)=\left(k_{4} z_{4}\left(t_{k}\right)+\widehat{f}_{4}\left(t_{k}\right)+\widehat{d}_{4}\left(t_{k}\right)\right), \\
z_{4}\left(t_{k}\right)=x_{4}\left(t_{k}\right)-x_{4}^{*}\left(t_{k}\right), \\
x_{4}^{*}\left(t_{k}\right)=\left(k_{3} z_{3}\left(t_{k}\right)+\widehat{f}_{3}\left(t_{k}\right)+\widehat{d}_{3}\left(t_{k}\right)\right), \\
z_{3}\left(t_{k}\right)=x_{3}\left(t_{k}\right)-x_{3}^{*}\left(t_{k}\right), \\
x_{3}^{*}\left(t_{k}\right)=\left(k_{2} z_{2}\left(t_{k}\right)+\widehat{f}_{2}\left(t_{k}\right)+\widehat{d}_{2}\left(t_{k}\right)\right), \\
z_{2}\left(t_{k}\right)=x_{2}\left(t_{k}\right)-x_{2}^{*}\left(t_{k}\right), \\
x_{2}^{*}\left(t_{k}\right)=\left(k_{1} z_{1}\left(t_{k}\right)+\widehat{f}_{1}\left(t_{k}\right)+\widehat{d}_{1}\left(t_{k}\right)\right), \\
z_{1}\left(t_{k}\right)=x_{1}\left(t_{k}\right)-y_{r}\left(t_{k}\right) .
\end{gathered}
$$

\section{Numerical Simulation Results}

In this section, in order to verify the control efficiency and superior disturbance attenuation of the proposed controller, three conventional methods including PI control method and domination feedback control method are chosen to be compared with the proposed method. The parameters of the MT system utilized in following simulations are listed in Table 1.

The lumped mismatched disturbances are settled as

$$
\begin{aligned}
& d_{1}(t)= \begin{cases}30, & 4<t \leq 8, \\
3 t, & 24<t \leq 32, \\
0, & \text { else, }\end{cases} \\
& d_{2}(t)=0.2, \\
& d_{3}(t)=-0.3, \\
& d_{4}(t)=0.5 .
\end{aligned}
$$

The desired speed curve $y_{r}$ is characterized by

$$
y_{r}= \begin{cases}0.1 t, & 0 \leq t \leq 10 \\ 0.8, & 20 \leq t<30 \\ 1, & \text { else. }\end{cases}
$$


TABLE 1: Parameters of MT system.

\begin{tabular}{lcc}
\hline Parameters & Values & Units \\
\hline Fuel actuator parameters & $K_{a}=1, T_{a}=0.4$ & Constant \\
Discharge lag time constant & $T_{\mathrm{CD}}=0.2$ & $\mathrm{~s}$ \\
Valve position parameters & $a=0.05, b=c=1$ & Constant \\
Inertia of rotor and load & $J=8 \times 10^{-4}$ & $\mathrm{~K}_{\mathrm{g}} \cdot \mathrm{m}^{2}$ \\
The electromagnetic torque & $T_{e}=0.2$ & $\mathrm{Nm}$ \\
Minimum load & $k_{l}=0.23$ & $\mathrm{~N}$ \\
Maximum fuel demand & $k_{f}=0.77$ & $\mathrm{~N}$ \\
\hline
\end{tabular}

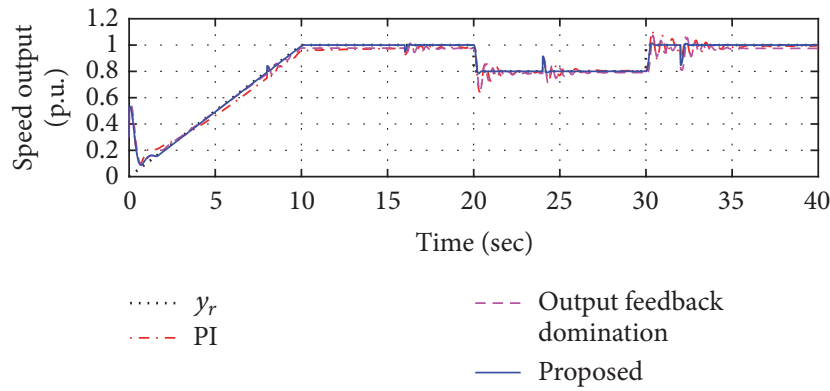

FIGURE 1: Speed tracking performance comparison of system (5) under three controllers: the proposed (solid line), domination (dashed line), and PI (dash-dot line).

Using output feedback domination method mentioned in paper [16], a control law for system (5) presented as

$$
\begin{aligned}
\dot{\vec{z}}_{1} & =\widehat{z}_{2}+L\left(y-\widehat{z}_{1}\right), \\
\dot{\vec{z}}_{2} & =\widehat{z}_{3}+L^{2}\left(y-\widehat{z}_{1}\right), \\
\dot{\vec{z}}_{3} & =\widehat{z}_{4}+L^{3}\left(y-\widehat{z}_{1}\right), \\
\dot{\vec{z}}_{4} & =v+L^{4}\left(y-\widehat{z}_{1}\right), \\
u & =v / g_{1} / g_{2} / g_{3} / g_{4}, \\
v & =-L b_{4}\left(\widehat{z}_{4}+L b_{3}\left(\widehat{z}_{3}+L b_{2}\left(\widehat{z}_{2}+L b_{1} \widehat{z}_{1}\right)\right)\right) .
\end{aligned}
$$

To guarantee the fairness of the comparison in numerical simulations, input voltage $u$ of the MT system should be bounded in the same level. The parameters for controller (46) are selected as $b_{1}=1, b_{2}=0.5, b_{3}=50, b_{4}=50$, and $L=60$. The control parameters for proposed method are selected as $k_{1}=10, k_{2}=15, k_{3}=15$, and $k_{4}=200$, while the observer parameters are selected as $\lambda_{1}=100, \lambda_{2}=100, \lambda_{3}=100$, and $\lambda_{4}=100$. The proportional parameter and integral parameter of PI controller are separately selected as 6 and 0.6.

From Figure 1, as a whole, it can be generally observed that the three controllers can all stabilize the MT system. In the aspect of the stabilization accuracy, the output feedback PI control method can take an acceptable regulation performance when the MT system is perturbed and presents a relative better tracking effect than the output feedback domination method, whereas, with adopting the domination feedback technique, fast response of the control systems (8)-(38) and

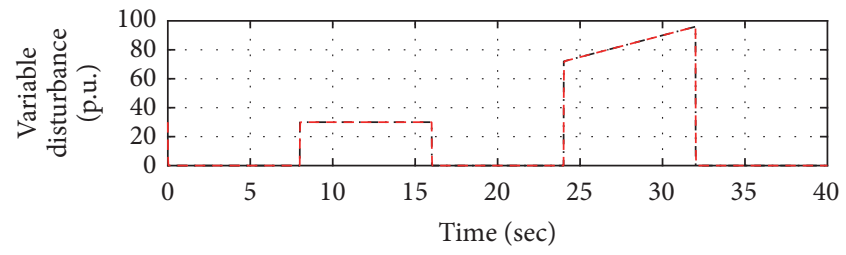

$$
\begin{aligned}
-\cdot & d_{1} \\
--- & \widehat{d}_{1}
\end{aligned}
$$

(a) Variable disturbance in speed-loop and its estimation

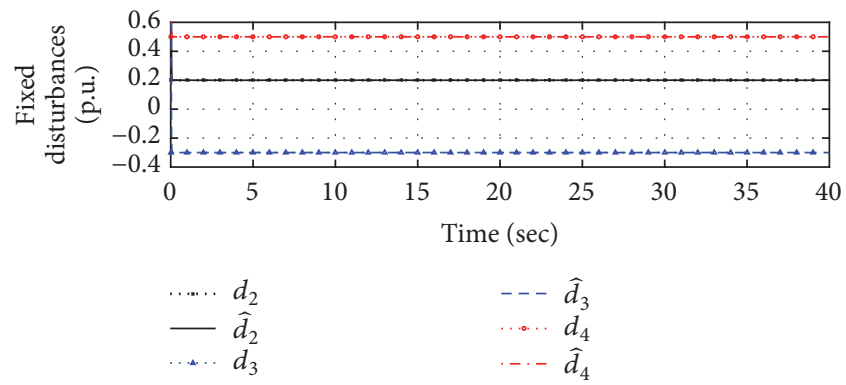

(b) Fixed disturbances in three other loops and their estimations

Figure 2: Disturbances and their estimations in control channels.

(46) provide benign response curves when control objective changes, while PI control requires a relative more time to be stable. Moreover, it can be noticed that applying an observer to the domination feedback controller provides a nice tracking investment without being impact from the variable and fixed unknown disturbances.

Figure 2 presents the time history of the lumped disturbances settled in system (5) and estimations of these three disturbances with the DO based domination control methodology. It can be clearly found that, with a disturbance observer, the disturbances can be precisely estimated by adding a disturbance observer and a feedforward part.

One can observe from Figure 3 that the control voltage of system (5) with three controllers is limited by a range $u \in[-1,1]$ p.u., which satisfies the control ability of the MT system.

\section{Conclusions}

In this paper, nonlinearities of the MT system are handled with the feedback domination approach, while the lumped 


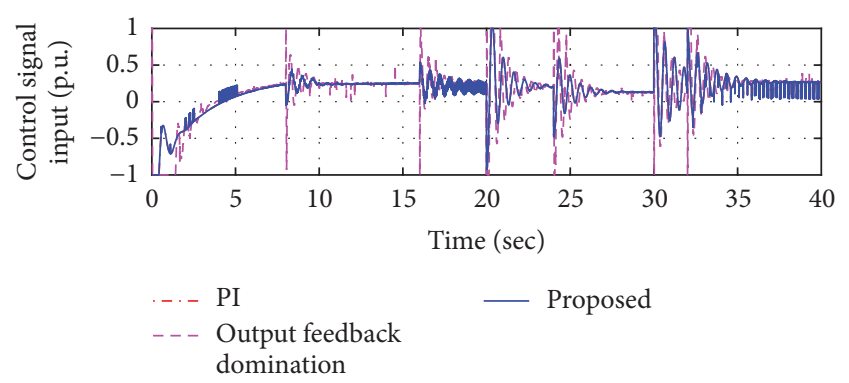

FIGURE 3: Control signals input of the MT with three controllers: the proposed (solid line), domination (dashed line), and PI (dashdot line).

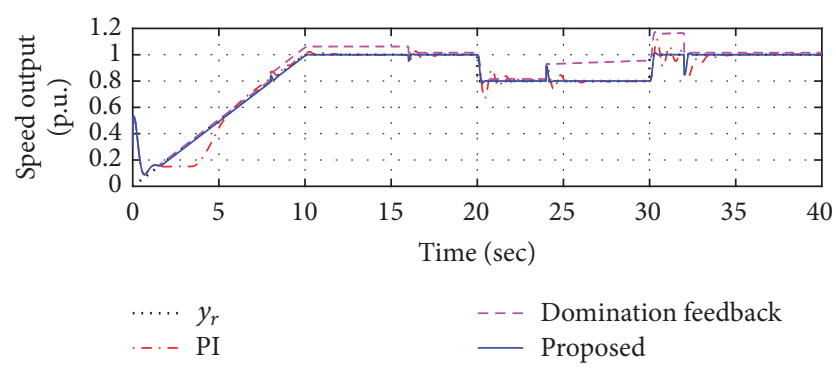

FIGURE 4: Speed tracking performance comparison of system (5) under three controllers: the proposed (solid line), domination (dashed line), and PI (dash-dot line).

disturbances are compensated in feedforward channels by employing a nonlinear disturbance observer. Hence a global tracking control scheme has been developed for the MT system with a simple control form. This novel method has been theoretically proved rigorously using the Lyapunov stability analysis. Numerical simulation results verify the closedloop performance and disturbance attenuation ability with comparison to PI control and output domination feedback control results.

\section{Appendix}

The proposed controller requires more information than compared ones in above simulations; hence in Figure 4, all controllers utilize the same physical messages.

The PI controller requires eight parameters: the proportional parameters of four loop controllers are separately selected as 9, 10, 50, and 0.0008 and the integral parameters of four loop controllers are separately selected as $2.5,0.5,4.5$, and 0.001 .

The domination feedback controller for system (5) is redesigned as

$$
\begin{aligned}
& z_{1}=x_{1}-y_{r}, \\
& \widehat{f}_{1}=f_{1}\left(y_{r}\right), \\
& x_{2}^{*}=-\frac{1}{g_{1}}\left(k_{1} z_{1}+\widehat{f}_{1}\right) ; \\
& z_{2}=x_{2}-x_{2}^{*},
\end{aligned}
$$

$$
\begin{aligned}
& \hat{f}_{2}=f_{2}\left(y_{r},-\frac{\hat{f}_{1}}{g_{1}}\right), \\
& x_{3}^{*}=-\frac{1}{g_{2}}\left(k_{2} z_{2}+\widehat{f}_{2}\right) ; \\
& z_{3}=x_{3}-x_{3}^{*}, \\
& \widehat{f}_{3}=f_{3}\left(y_{r},-\frac{\hat{f}_{1}}{g_{1}},-\frac{\widehat{f}_{2}}{g_{2}}\right), \\
& x_{4}^{*}=-\frac{1}{g_{3}}\left(k_{3} z_{3}+\widehat{f}_{2}\right) ; \\
& z_{4}=x_{4}-x_{4}^{*}, \\
& \widehat{f}_{4}=f_{4}\left(y_{r},-\frac{\hat{f}_{1}}{g_{1}},-\frac{\hat{f}_{2}}{g_{2}},-\frac{\widehat{f}_{3}}{g_{3}}\right), \\
& u=-\frac{1}{g_{4}(x)}\left(k_{4} z_{4}+\widehat{f}_{4}\right),
\end{aligned}
$$

where $k_{1}=10, k_{2}=15, k_{3}=15$, and $k_{4}=200$.

With this comparison, one can also explain the phenomenon appearing in Figure 1, which indicates the proposed method behaves with a better steady stable performance than domination feedback control method without a DO technique.

\section{Conflicts of Interest}

The authors declare that they have no conflicts of interest.

\section{Acknowledgments}

This work is supported by National Natural Science Foundation of China (Grant nos. 51705304, 51607111) and Natural Science Foundation of Shanghai (Grant no. 16ZR1413400).

\section{References}

[1] A. Bertani, C. Bossi, F. Fornari, S. Massucco, S. Spelta, and F. Tivegna, "A microturbine generation system for grid connected and islanding operation," in Proceedings of the 2004 IEEE PES Power Systems Conference and Exposition, pp. 360-365, usa, October 2004.

[2] D. Thomas, O. Deblecker, A. Bagheri, and C. S. Ioakimidis, "A scheduling optimization model for minimizing the energy demand of a building using electric vehicles and a microturbine," in Proceedings of the 2nd IEEE International Smart Cities Conference, ISC2 2016, Italy, September 2016.

[3] U. Siddaraj and S. Tangi, "Integration of DG systems composed of photovoltaic and a micro-turbine in remote areas," in Proceedings of the 5th International Conference on Computation of Power, Energy Information and Communication, ICCPEIC 2016, pp. 828-831, India, April 2016.

[4] X. Xu, H. Jia, H.-D. Chiang, D. C. Yu, and D. Wang, "Dynamic modeling and interaction of hybrid natural gas and electricity supply system in microgrid," IEEE Transactions on Power Systems, vol. 30, no. 3, pp. 1212-1221, 2015. 
[5] S. R. Guda, C. Wang, and M. H. Nehrir, "A simulink-based microturbine model for distributed generation studies," in Proceedings of the 37th Annual North American Power Symposium, 2005, pp. 269-274, USA, October 2005.

[6] A. K. Saha, S. Chowdhury, S. P. Chowdhury, and P. A. Crossley, "Modeling and performance analysis of a microturbine as a distributed energy resource," IEEE Transactions on Energy Conversion, vol. 24, no. 2, pp. 529-538, 2009.

[7] X. Tan, X. Su, K. Zhao, and M. Tan, "Robust adaptive backstepping control of micro-turbines," in Proceedings of the 28th Chinese Control and Decision Conference, CCDC 2016, pp. 490493, China, May 2016.

[8] N. S. Sisworahardjo, M. Y. El-Sharkh, and M. S. Alam, "Neural network controller for microturbine power plants," Electric Power Systems Research, vol. 78, no. 8, pp. 1378-1384, 2008.

[9] Z. Guo and K. Y. Lee, "A self-adaptive fuzzy PI controller of power conditioning system for hybrid fuel-cell/turbine power plant," in Proceedings of the 43rd North American Power Symposium, NAPS 2011, USA, August 2011.

[10] M. Zhu, X. Wu, Y. Li, J. Shen, and J. Zhang, "Modeling and model predictive control of micro gas turbine-based combined cooling, heating and power system," in Proceedings of the 28th Chinese Control and Decision Conference, CCDC 2016, pp. 6570, China, May 2016.

[11] J. Q. Han, "From PID to active disturbance rejection control," IEEE Transactions on Industrial Electronics, vol. 56, no. 3, pp. 900-906, 2009.

[12] W.-H. Chen, "Disturbance observer based control for nonlinear systems," IEEE/ASME Transactions on Mechatronics, vol. 9, no. 4, pp. 706-710, 2004.

[13] C. Zhang, J. Yang, S. Li, and N. Yang, "A generalized active disturbance rejection control method for nonlinear uncertain systems subject to additive disturbance," Nonlinear Dynamics, vol. 83, no. 4, pp. 2361-2372, 2016.

[14] T. Yu and J.-P. Tong, "Auto disturbance rejection control of microturbine system," in Proceedings of the IEEE Power and Energy Society 2008 General Meeting: Conversion and Delivery of Electrical Energy in the 21st Century, PES, USA, July 2008.

[15] J. Yang, H. Liu, S. Li, and X. Chen, "Nonlinear disturbance observer based robust tracking control for a PMSM drive subject to mismatched uncertainties," in Proceedings of the 31st Chinese Control Conference, CCC 2012, pp. 830-835, chn, July 2012.

[16] C. Qian and W. Lin, "Output feedback control of a class of nonlinear systems: a nonseparation principle paradigm," Institute of Electrical and Electronics Engineers Transactions on Automatic Control, vol. 47, no. 10, pp. 1710-1715, 2002.

[17] X. Niu, C. Zhang, and H. Li, "Active disturbance attenuation control for permanent magnet synchronous motor via feedback domination and disturbance observer," IET Control Theory \& Applications, vol. 11, no. 6, pp. 807-815, 2017.

[18] H. K. Khalil, Noninear Systems, Prentice-Hall, NJ, USA, 1996.

[19] C. Qian and W. Lin, "A continuous feedback approach to global strong stabilization of nonlinear systems," Institute of Electrical and Electronics Engineers Transactions on Automatic Control, vol. 46, no. 7, pp. 1061-1079, 2001. 


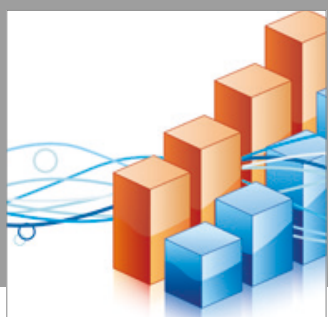

Advances in

Operations Research

vatersals

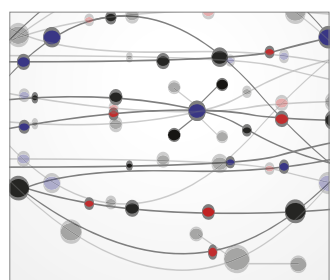

\section{The Scientific} World Journal
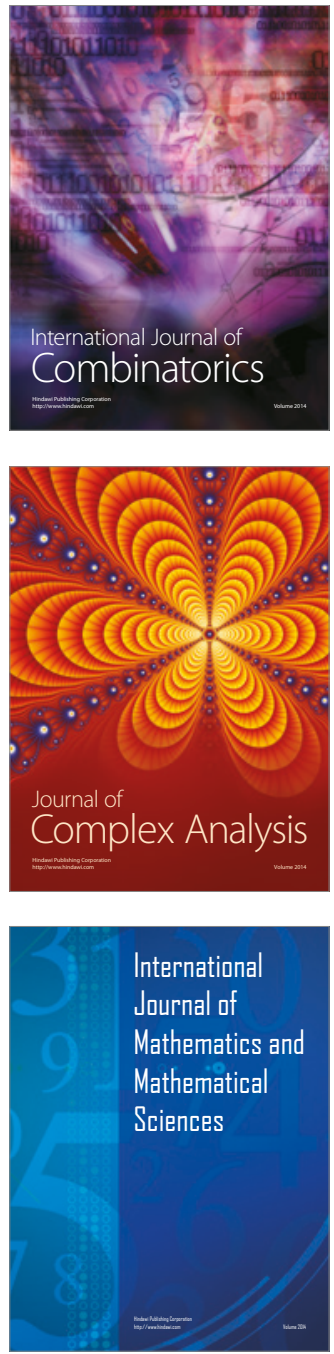
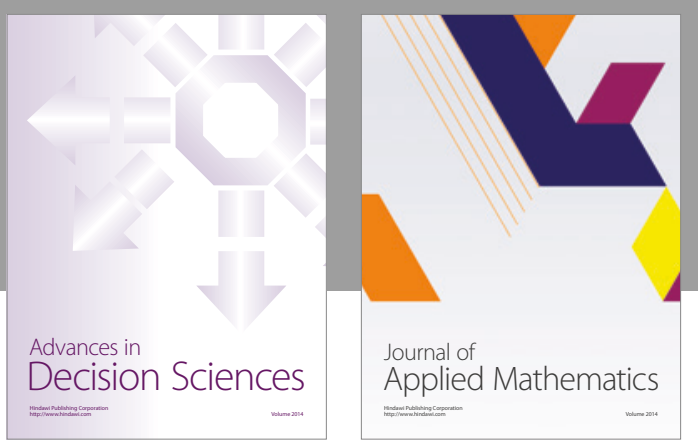

Algebra

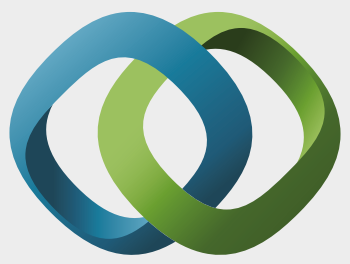

\section{Hindawi}

Submit your manuscripts at

https://www.hindawi.com
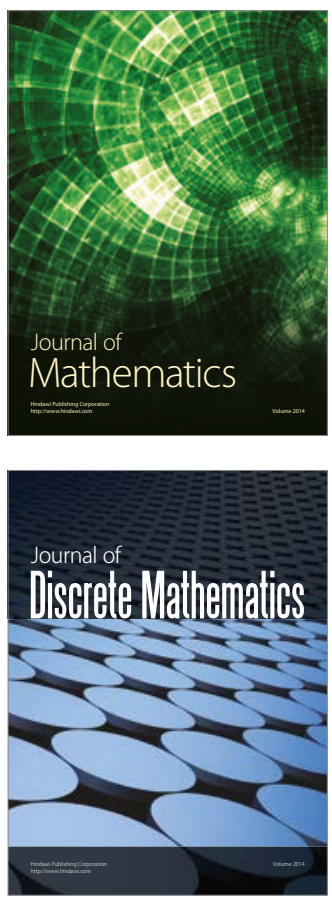

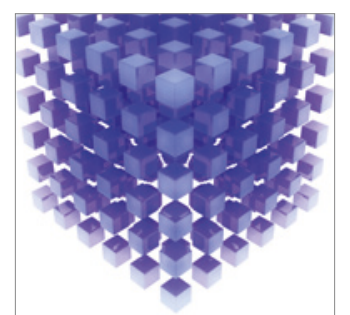

Mathematical Problems in Engineering
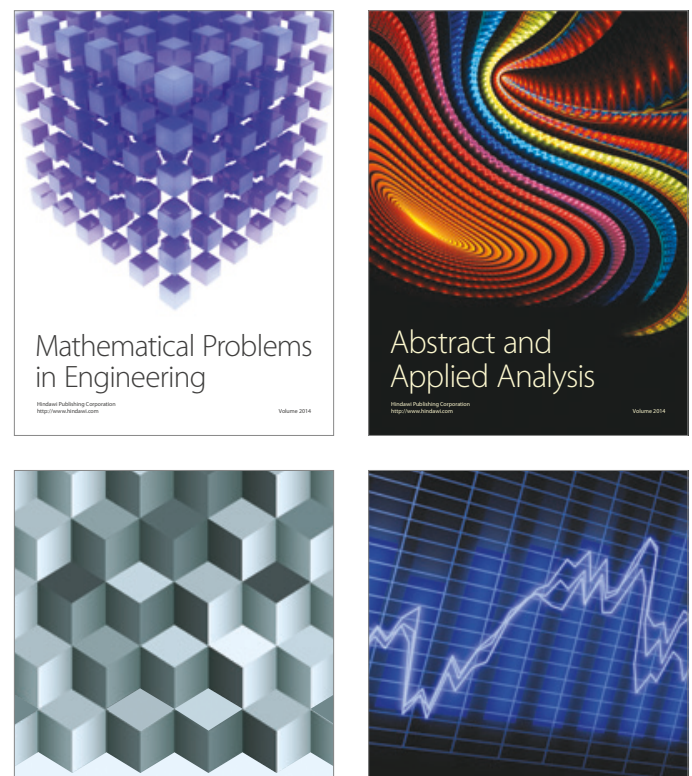

Journal of

Function Spaces

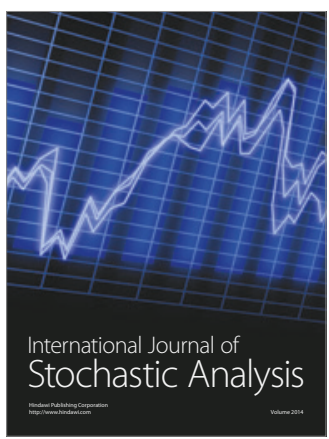

Probability and Statistics
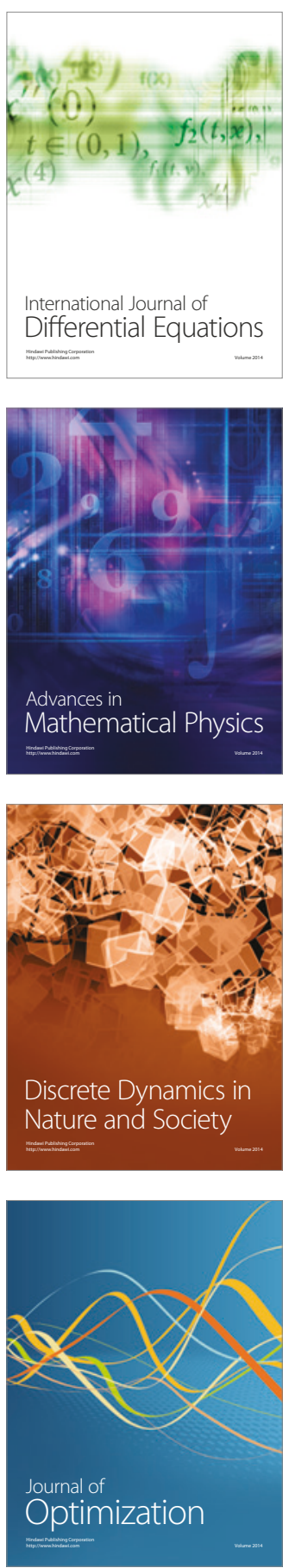